\title{
The relationship between the evolution of microRNA targets and the length of their UTRs
}

\author{
Chao Cheng ${ }^{1}$, Nitin Bhardwaj ${ }^{1}$ and Mark Gerstein ${ }^{* 1,2,3}$
}

\author{
Address: ${ }^{1}$ Program in Computational Biology and Bioinformatics, Yale University, New Haven, CT 06520, USA, ${ }^{2}$ Department of Molecular \\ Biophysics and Biochemistry, Yale University, New Haven, CT 06520, USA and 'Department of Computer Science, Yale University, New Haven, \\ CT 06520, USA \\ Email: Chao Cheng - chao.cheng@yale.edu; Nitin Bhardwaj - nitin.bhardwaj@yale.edu; Mark Gerstein* - Mark.Gerstein@yale.edu \\ * Corresponding author
}

Published: 14 September 2009

BMC Genomics 2009, 10:431 doi:10.1|86/|47|-2|64-|0-43|
Received: 2 February 2009

Accepted: 14 September 2009

This article is available from: http://www.biomedcentral.com/|47|-2/64//0/43 I

(C) 2009 Cheng et al; licensee BioMed Central Ltd.

This is an Open Access article distributed under the terms of the Creative Commons Attribution License (http://creativecommons.org/licenses/by/2.0), which permits unrestricted use, distribution, and reproduction in any medium, provided the original work is properly cited.

\begin{abstract}
Background: MicroRNAs (miRNAs) are endogenous small RNA molecules that modulate the gene expression at the post-transcription levels in many eukaryotic cells. Their widespread and important role in animals is gauged by estimates that $\sim 25 \%$ of all genes are miRNA targets.

Results: We perform a systematic investigation of the relationship between miRNA regulation and their targets' evolution in two mammals: human and mouse. We find genes with longer 3' UTRs are regulated by more distinct types of miRNAs. These genes correspondingly tend to have slower evolutionary rates at the protein level. Housekeeping genes are another class of genes that evolve slowly. However, they have a distinctly different type of regulation, with shorter 3'UTRs to avoid miRNA targeting.

Conclusion: Our analysis suggests a two-way evolutionary mechanism for miRNA targets on the basis of their cellular roles and the length of their 3' UTRs. Functionally critical genes that are spatially or temporally expressed are stringently regulated by miRNAs. While housekeeping genes, however conserved, are selected to have shorter 3'UTRs to avoid miRNA regulation.
\end{abstract}

\section{Background}

Regulation of gene expression at the transcriptional level plays a central role in governing all cellular activities. However, the significance of gene regulation at the posttranscriptional level has gained a lot of interest and popularity over the last decade. MicroRNAs (miRNAs) are one of the two regulators, along with siRNA (short interfering RNAs), that have emerged as important players in posttranslation regulation and mRNA decay. miRNAs are endogenously expressed small RNAs that regulate gene expression at the post-transcriptional level $[1,2]$. To repress expression of mRNAs, miRNAs recognize target sites in their 3' un-translated region (3'UTR) via base pair- ing, leading to their degradation or inhibition of their translation [3-5]. Considering the critical roles of miRNAs in gene expression regulation [6-8], it would be interesting and insightful to investigate the regulatory effect of miRNAs from an evolutionary perspective.

Intuitively, for functionally important proteins that contribute significantly to individual fitness, selection pressure may exhibit its effect in two aspects. On one hand, non-synonymous mutations that lead to slightly deleterious substitutions accumulate slowly in these proteins [9]. On the other hand, expression of genes encoding these proteins is subjected to delicate but robust regulation at 
the transcriptional and post-transcriptional levels. Therefore, genes under more stringent regulation by miRNAs are expected to evolve more slowly at the protein level. In this study, we investigated the relationship between miRNA regulation and protein evolutionary rate in two mammals: human and mouse. For these two species, a large number of miRNAs have been identified that enables a systematic analysis with statistically significant conclusions.

\section{Results and Discussion \\ Protein evolutionary rates are negatively correlated with the number of regulatory miRNAs}

First, we calculated the number of distinct regulatory miRNAs for each human and mouse gene based on the predicted miRNA binding sites by the PITA algorithm [10]. We chose PITA for miRNA target prediction because it has been shown to achieve high prediction accuracy, and more importantly, it takes advantage of the target accessibility but not conservation information (used by most of the other methods) to reduce false positives [10]. Such an omission is important for this study because we find that the conservation at 3'UTR is correlated with the conservation at the coding regions as well as the protein evolutionary rate, and therefore it may complicate our analysis. Specifically, we calculated the average conservation score of 3'UTR and coding regions for all human mRNAs according to the sequence alignment of 17 vertebrate species [11]. The results indicate that the conservation score at 3'UTR is positively correlated with that at the coding region ( $\rho=0.55$ ) and negatively correlated with the human protein evolutionary rate $\mathrm{Ka} / \mathrm{Ks}$ against mouse ( $\rho$ $=-0.43)$. The second reason for using accessibility over conservation information is that previous experiments have shown that, in addition to conserved miRNAs target sites, non-conserved sites are also functional and mediate repression $[12,13]$.

Next, using the homologous pairs between mouse and human from HomoloGene [14], we obtained the evolutionary rates of human and mouse proteins. Evolutionary rate for protein is defined as the $\mathrm{Ka} / \mathrm{Ks}$ ratio, where $\mathrm{Ka}$ and Ks are the rates of non-synonymous and synonymous substitutions, respectively. Finally, for those genes for which we had both miRNA target site predictions and evolutionary information, we calculated the correlation between the number of distinct regulatory miRNAs and the protein's evolutionary rates. Our results indicate a significant negative correlation between the two in both human (Spearman correlation coefficient, $\rho=-0.21, \mathrm{P}=$ $7 \mathrm{E}-128$ when the $\mathrm{Ka} / \mathrm{Ks}$ ratios are obtained by aligning against homologous proteins in mouse; Figure 1a) and mouse $(\rho=-0.21, P=2 \mathrm{E}-139$ when the $\mathrm{Ka} / \mathrm{Ks}$ ratios are obtained using human as the reference; Figure 1b), suggesting that genes regulated by more distinct miRNAs at the 3'UTR regions tend to have slower evolutionary rates. Similar trends were obtained when other species ranging from chicken to human are used as references to obtain the evolutionary rates (Figure 1c). We also performed the same analysis using two other miRNA target site prediction methods: miRanda and TargetScan [15-19]. For the reasons stated above, we did not impose conservation filtering but kept all the potential miRNA target sites in our analysis. Again, our results indicate a significant negative correlation between the number of regulatory miRNAs and protein evolutionary rate in both human $(\rho=-0.17, P$ $=5 \mathrm{E}-70$ when the $\mathrm{Ka} / \mathrm{Ks}$ ratios are obtained using mouse as the reference with miRanda; $\rho=-0.187, \mathrm{P}=2 \mathrm{E}-80$ with TargetScan) and mouse $(\rho=-0.11, \mathrm{P}=2 \mathrm{E}-15$ when the $\mathrm{Ka} /$ Ks ratios are obtained using human as the reference; see Additional file 1: Figure S1). This demonstrates that our results are robust to the way protein evolutionary rates are obtained and to the miRNA target site prediction method (see Additional file 2: Table S1 and Additional file 3, 4, 5 \&6: Figure S2-S5).

\section{The negative correlation is independent of the expression intensities of miRNA targets}

It can be argued that the intensity of gene expression, which relates inversely to the rate of protein sequence evolution [20], could be the underlying cause of the negative correlation between number of regulatory miRNAs and evolutionary rate. To rule out this possibility, we calculated the average expression intensities of human genes in 79 tissues [21]. Our results indicate a negative correlation between average expression level of human genes and their evolutionary rates $(\rho=-0.18, \mathrm{P}=7 \mathrm{E}-70$ when the $\mathrm{Ka} /$ Ks ratios are obtained using mouse as the reference). However, there is no significant correlation between the number of regulatory miRNAs and gene expression intensities ( $\rho=-0.016, P=0.12)$. Therefore, the negative correlation between the number of regulatory miRNAs and the protein's evolutionary rate is unlikely to be mediated by gene expression intensities. This argument is further validated by parametric $(-0.20, \mathrm{P}=1 \mathrm{E}-85)$ and non-parametric $(-0.20, P=2 \mathrm{E}-86)$ partial correlation coefficients between the number of regulatory miRNAs and evolutionary rate with the expression intensity being held constant [22].

\section{Genes with longer 3'UTR tend to evolve at slower rates}

In general, genes with longer 3'UTRs are likely to be regulated by more miRNAs. We therefore examine the correlation between 3'UTR length and evolutionary rate. As expected, we find that there is a negative correlation between these two in both human $(\rho=-0.17, \mathrm{P}=6 \mathrm{E}-72$ when the $\mathrm{Ka} / \mathrm{Ks}$ ratios are obtained using mouse as the reference, Figure $2 \mathrm{a})$ and mouse $(\rho=-0.11, \mathrm{P}=7 \mathrm{E}-17$ when the $\mathrm{Ka} / \mathrm{Ks}$ ratios are obtained using human as the reference, Figure 2b). Similar results were obtained using 
(a)

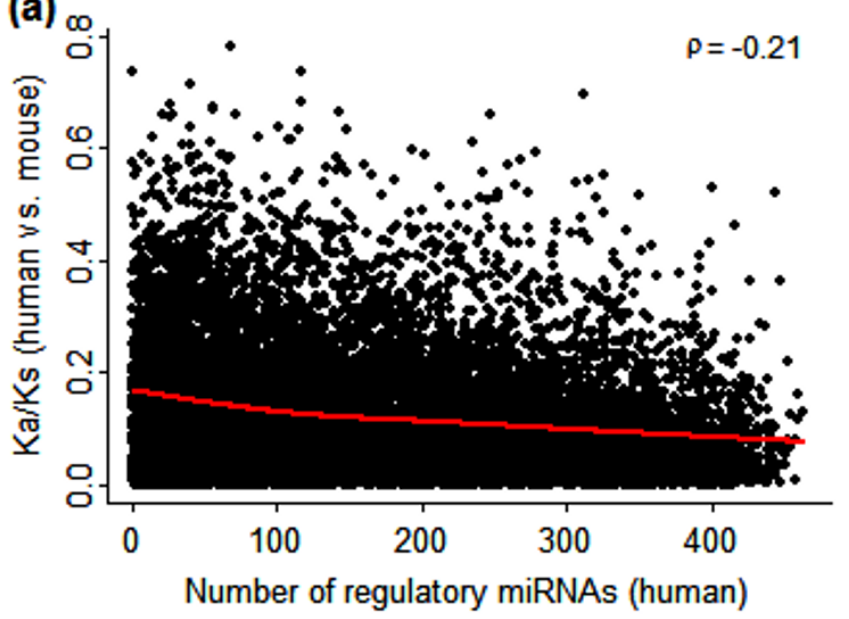

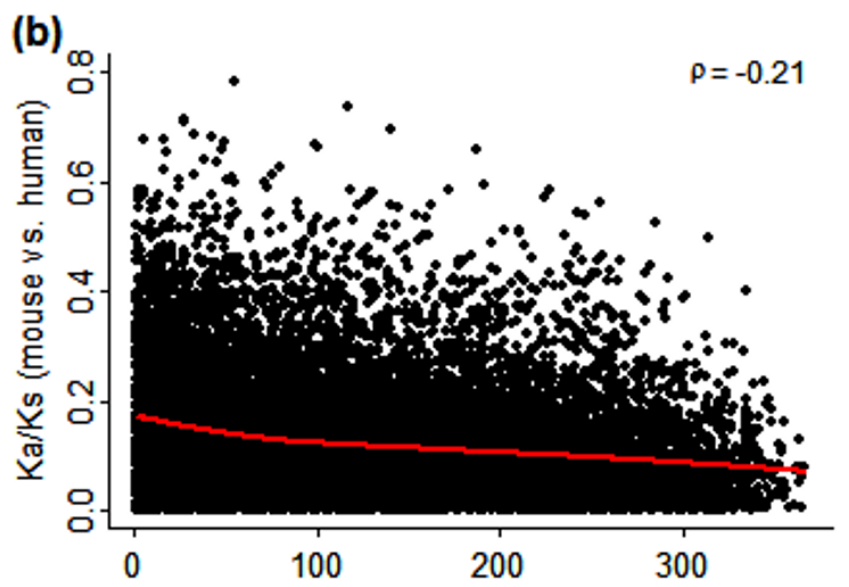

Number of regulatory miRNAs (mouse)

(c)

\begin{tabular}{llll|llll}
\hline \hline Human vs. & Number of genes & Correlation & P-value & Mouse vs. & Number of genes & Correlation & P-value \\
\hline chimpanzee & 11326 & -0.11 & $2 . E-32$ & human & 14579 & -0.21 & $2 . E-139$ \\
mouse & 13280 & -0.21 & $7 . E-128$ & chimpanzee & 12188 & -0.21 & $6 . E-121$ \\
rat & 12270 & -0.20 & $4 . E-107$ & rat & 13909 & -0.21 & $1 . E-140$ \\
cow & 11683 & -0.21 & $8 . E-115$ & cow & 12448 & -0.18 & $1 . E-93$ \\
chicken & 8061 & -0.18 & $1 . E-57$ & chicken & 7830 & -0.17 & $1 . E-53$ \\
\hline
\end{tabular}

\section{Figure I}

The relation between the number of regulatory miRNAs and the protein evolutionary rate. (a, b) The evolutionary rates of proteins in human (a) and mouse (b) are estimated as the $\mathrm{Ka} / \mathrm{Ks}$ ratios using the other organism as reference. The number of regulatory miRNAs is calculated by counting miRNAs that have at least one target site within the 3'UTR of a gene. The red lines show the smoothed relation between the two quantities estimated by LOESS method. $\rho$ indicates Spearman correlation coefficient. (c) Correlation between the number of regulatory miRNAs and protein evolution rate using various species as references. miRNA binding sites are based on PITA predictions.

miRNA targets predicted by the miRanda and TargetsScan algorithms (see Additional file 7: Table S2).

Previous studies, however, have shown that housekeeping genes are likely to have shorter 3'UTRs to avoid miRNA regulation, suggesting that they may have a different scenario in terms of miRNA regulation and protein evolution $[23,24]$. So, we compared the human housekeeping [23] with non-housekeeping genes and found that housekeeping genes are more likely to have slower evolutionary rates, shorter 3'UTRs and less number of regulatory miRNAs (Figure 2c). Interestingly, a recent study demonstrated the increased relative expression of the mRNA isoforms with shortened 3'UTR and fewer miRNA target sites in proliferating cells, suggesting that modulating 3'UTR length through alternative splicing is likely to be a biological mechanism to adjust miRNA regulation [25]. However, it should be noted here that while miRNAs overall target longer UTRs, the number of target sites does not simply scale with the length; rather, target sites are preferentially found towards the end of the UTRs [26-28].
Correlation between number of miRNAs and evolutionary rate is beyond the length of the 3'UTR region

We wanted to examine if the negative correlation between evolutionary rate and number of regulating miRNAs goes beyond the length of the 3'UTR region. So, we integrated a control for the length bias and found that there is no significant correlation between the density of miRNA binding and the protein evolutionary rate $(\omega)$, which may indicate that change of the number of regulatory miRNAs for genes is mainly achieved by change of its 3'UTR length and requires no change of binding site density. However, the correlation between the number of miRNAs $(\mathrm{N})$ and protein evolutionary rate $(\omega)$ cannot be fully explained by 3'UTR length (L) as indicated by partial correlation coefficients: for human $\rho(\omega, N \mid L)=-0.172(P=1 E-60)$ when PITA is used; $\rho(\omega, \mathrm{N} \mid \mathrm{L})=-0.151(\mathrm{P}=6 \mathrm{E}-53)$ when TargetScan is used. On the other hand, the correlation between $\omega$ and $\mathrm{L}$ is largely explained by $\mathrm{N}$ : $\rho(\omega, \mathrm{L} \mid \mathrm{N})=$ $0.044(\mathrm{P}=3 \mathrm{E}-5)$ when PITA is used and $\rho(\omega, \mathrm{L} \mid \mathrm{N})=$ $0.048(\mathrm{P}=1 \mathrm{E}-6)$ when TargetScan is used. These results suggest that anti-correlation between evolutionary rates 
(a)

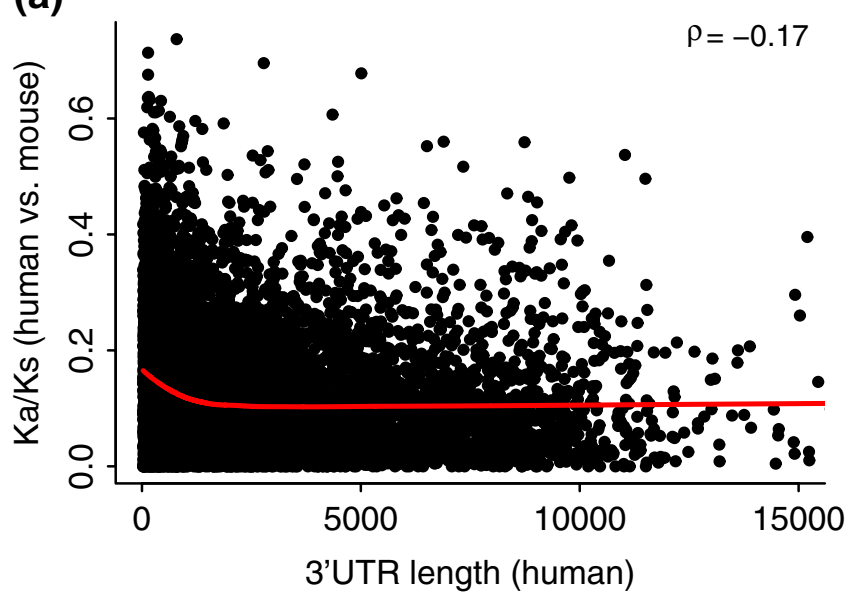

(b)

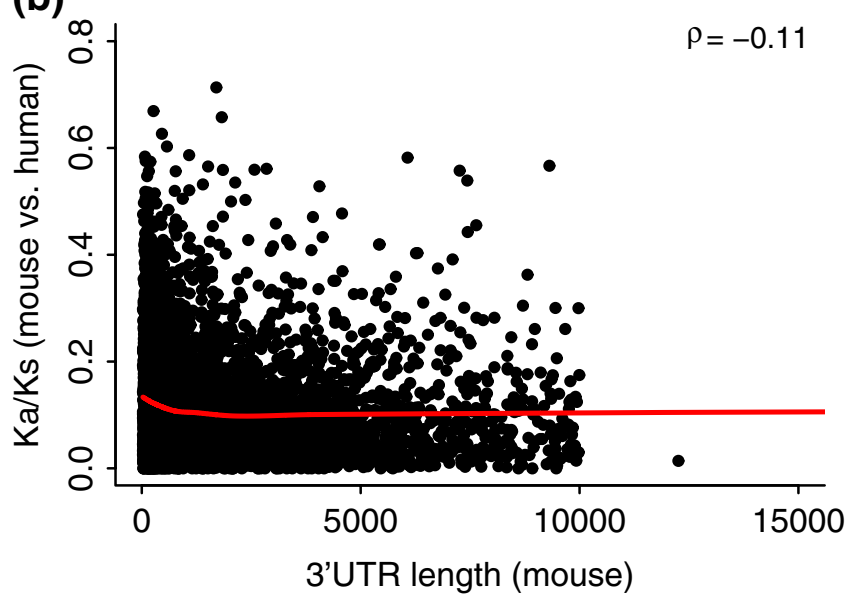

(c)
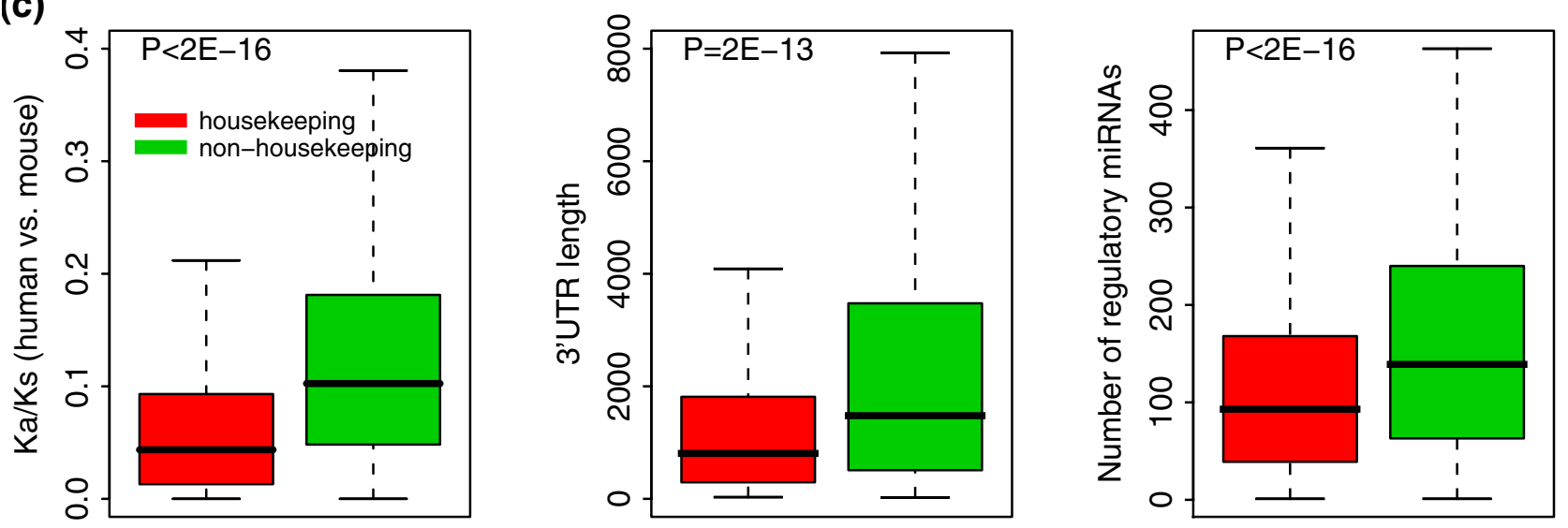

\section{Figure 2}

The relation between protein evolutionary rate and 3'UTR length of mRNAs. (a, b) Global inverse relationship between protein evolutionary rate and 3'UTR length for human (a) and mouse (b) mRNAs. (c) Difference between housekeeping (red) and non-housekeeping (green) human genes in evolutionary rate (Left), 3'UTR length (Middle) and the number of regulatory miRNAs (Right). The P-values are calculated using the Wilcoxon rank sum test.

and number of miRNAs is not mediated by the 3'UTR length.

We further show that the correlation between protein evolution and the number of miRNA binding sites is mediated by functional miRNA binding sites in the 3'UTR region. We do so by generating shuffled miRNAs with permuted nucleotide sequences while keeping the length and base composition unchanged in human. We predicted targets of miRNAs in a similar way as TargetScan - searching for the presence of 8 mer (exact match to positions 2-8 of the mature miRNA followed by an 'A'), 7 mer-m8 (exact match to positions 2-8 of the mature miRNA) and 7 mer1A (exact match to positions 2-7 of the mature miRNA followed by an 'A') sites that match the seed region of each
miRNA. Our results indicate a significant correlation between the number of shuffled miRNAs for a gene and the protein evolution rate (computed as $\mathrm{Ka} / \mathrm{Ks}$ against mouse), which is expected due to the strong correlation between 3'UTR length and the number of shuffled miRNA binding sites. However, after taking into account the 3'UTR length, the correlation between them is abolished as shown by the partial correlation $\rho(\omega, \mathrm{N} \mid \mathrm{L})=0.014(\mathrm{P}$ $>0.05$ ) indicating that correlation between evolutionary rate and the number of shuffled miRNA binding sites can be fully explained by 3'UTR length.

\section{Correlation of genetic features with evolutionary rate}

We also determined the correlation of protein evolutionary rate (Ka/Ks ratio) with 5 gene features: 5'UTR length, 
CDS length, cDNA length, first exon length and first intron length. All these features have negative correlation with $\mathrm{Ka} / \mathrm{Ks}$ ratios showing that more conserved proteins demonstrate higher values of the above features. But a more careful investigation indicated that this anti-correlation is due to the correlation between these features with 3'UTR length. There is only small (with the exception of first intron length) yet moderately significant correlation between them and the protein evolutionary rate after taking 3'UTR length into account as indicated by their partial correlations: -0.058 for 5 'UTR, -0.026 for CDS, -0.032 for cDNA, -0.033 for first exon and -0.10 for first intron lengths, respectively. On the other hand, after taking into account these features, the partial correlation between 3 'UTR length and $\mathrm{Ka} / \mathrm{Ks}$ ratio is still considerable: -0.16, $0.16,-0.10,-0.16$ and -0.15 when the 5 'UTR, CDS, cDNA, first exon and first intron lengths are held constant, respectively. Therefore, it seems that more conserved proteins tend to have longer 3'UTRs and first introns. The correlation between first intron length and protein conservation is interesting and indicates that factors other than miRNA regulation also shape protein evolution.

\section{Conclusion}

To understand how natural selection has shaped the evolution of miRNAs and their target genes, some past exploratory studies have been performed but they all have focused on the evolution of miRNAs or their target sites in 3 'UTR region $[12,13,24,29,30]$. In this novel study, we investigated how miRNA regulation is correlated with the evolution of proteins in human and mouse. Our results suggest that a two-way strategy has been implemented in mammals to achieve stringent regulation of genes at posttranscriptional level by miRNAs. First, functionally critical genes that are spatially or temporally expressed (nonhousekeeping genes) are stringently regulated by miRNAs. For robust regulation of these genes, longer 3'UTRs are preferred so that more target sites of distinct regulatory miRNAs can be included. Secondly, housekeeping genes, however conserved, are selected to have shorter 3'UTRs to avoid miRNA regulation.

\section{Methods}

miRNA target prediction data by PITA [10] was downloaded from http://genie.weizmann.ac.il/pubs/mir07/ mir07 data.html. The data contained binding information for 475 human and 375 mouse miRNAs at the 3'UTR regions of mRNAs for the two species. To predict miRNA targets using the miRanda method [16], we downloaded the human and mouse 3'UTR sequences from the PolyA Cleavage Site and 3'-UTR Database [31] which is available at http://harlequin.jax.org/pacdb/data.php. To ensure prediction accuracy, only 3'UTR sequences labeled with "very high confidence" in the database were included in our analysis. An mRNA could correspond to multiple
3'UTR sequences due to alternative splicing and in such cases the longest sequence was used.

The evolutionary rate for human and mouse proteins, measured by $\mathrm{Ka} / \mathrm{Ks}$ ratio, were calculated based on data from the HomoloGene database [14] available at http:// www.ncbi.nlm.nih.gov/homologene. The phastCons score, which is a measure of evolutionary conservation in 17 vertebrates, was downloaded from the UCSC Genome Browser at http://genome.ucsc.edu/. To calculate the conservation score for the 3'UTR or coding region of a specific mRNA, the phastCons scores of all nucleotides within it were averaged. Human housekeeping and non-housekeeping genes are categorized based previous work by Eisenberg et al. [23]. All calculations and analyses are performed using the R platform.

\section{Authors' contributions}

CC conceived of the study, carried out the data analysis and helped in drafting the manuscript. NB participated in the design of the study and helped in drafting the manuscript. MG conceived of the study, and participated in its design and coordination and helped to draft the manuscript. All authors read and approved the final manuscript.

\section{Additional material}

\section{Additional file 1}

Figure S1: The relation between number of regulatory miRNAs predicted by miRanda method and protein evolutionary rate in human (A) and mouse (B). Both Pearson correlation coefficient (PCC) and Spearman correlation coefficient (SCC) are shown.

Click here for file

[http://www.biomedcentral.com/content/supplementary/14712164-10-431-S1.eps]

\section{Additional file 2}

Table S1: correlation between the number of regulatory miRNAs predicted by miRanda method and protein evolutionary rate using various species as references. Correlation shown in the table is Spearman correlation coefficient. Click here for file

[http://www.biomedcentral.com/content/supplementary/14712164-10-431-S2.xls]

\section{Additional file 3}

Figure S2: The relation between number of regulatory human miRNAs predicetd by miRanda method and protein evolutionary rate using chimpanzee (A), rat (B), cow (C), and chicken (D) as reference, respectively. The number of regulatory miRNAs is calculated by counting miRNAs that have at least one target site within the 3'UTR of a gene. The red lines show the smoothed relation between evolutionary rate and number of regulatory miRNAs estimated by LOESS method. PCC and SCC represent Pearson correlation coefficient and Spearman correlation coefficient, respectively.

Click here for file

[http://www.biomedcentral.com/content/supplementary/14712164-10-431-S3.eps] 


\section{Additional file 4}

Figure S3: The relation between number of regulatory mouse miRNAs prediceted by miRanda method and protein evolutionary rate using human (A), chimpanzee (B), cow (C), and chicken (D) as reference, respectively. Figure legends are similar to those in Additional file 3: Figure $S 2$.

Click here for file

[http://www.biomedcentral.com/content/supplementary/1471-

2164-10-431-S4.eps]

\section{Additional file 5}

Figure S4: The relation between number of regulatory human miRNAs predicted by PITA method and protein evolutionary rate using chimpanzee (A), rat (B), cow (C), and chicken (D) as reference, respectively. Figure legends are similar to those in Additional file 3: Figure $S 2$.

Click here for file

[http://www.biomedcentral.com/content/supplementary/14712164-10-431-S5.eps]

\section{Additional file 6}

Figure S5: The relation between number of regulatory mouse miRNAs predicted by PITA method and protein evolutionary rate using human $(A)$, chimpanzee (B), cow (C), and chicken (D) as reference, respectively. Figure legends are similar to those in Additional file 3: Figure S2. Click here for file

[http://www.biomedcentral.com/content/supplementary/14712164-10-431-S6.eps]

\section{Additional file 7}

Table S2: Correlation between 3'UTR length of mRNAs and protein evolutionary rate using various species as references. Correlation shown in the table is Spearman correlation coefficient.

Click here for file

[http://www.biomedcentral.com/content/supplementary/14712164-10-431-S7.xls]

\section{Acknowledgements}

We acknowledge support from the $\mathrm{NIH}$ and from the AL Williams Professorship funds.

\section{References}

I. Ambros V: The functions of animal microRNAs. Nature 2004, 43 I (7006):350-355.

2. Lai EC: Micro RNAs are complementary to 3' UTR sequence motifs that mediate negative post-transcriptional regulation. Nat Genet 2002, 30(4):363-364.

3. Valencia-Sanchez MA, Liu J, Hannon GJ, Parker R: Control of translation and mRNA degradation by miRNAs and siRNAs. Genes Dev 2006, 20(5):5।5-524.

4. Bagga S, Bracht J, Hunter S, Massirer K, Holtz J, Eachus R, Pasquinelli $A E$ : Regulation by let-7 and lin- 4 miRNAs results in target mRNA degradation. Cell 2005, I 22(4):553-563.

5. Humphreys DT, Westman BJ, Martin DI, Preiss T: MicroRNAs control translation initiation by inhibiting eukaryotic initiation factor 4E/cap and poly(A) tail function. Proc Natl Acad Sci USA 2005, I 02(47): 1696I-16966.

6. Lewis BP, Burge CB, Bartel DP: Conserved seed pairing, often flanked by adenosines, indicates that thousands of human genes are microRNA targets. Cell 2005, I 20(I): I5-20.

7. Carrington JC, Ambros V: Role of microRNAs in plant and animal development. Science 2003, 30 I (563 I):336-338.
8. Kloosterman WP, Plasterk RH: The diverse functions of microRNAs in animal development and disease. Dev Cell 2006, I I (4):44I-450.

9. Hirsh $\mathrm{AE}$, Fraser HB: Protein dispensability and rate of evolution. Nature 200I, 4I I(684I): I046-I049.

10. Kertesz M, lovino N, Unnerstall U, Gaul U, Segal E: The role of site accessibility in microRNA target recognition. Nat Genet 2007, 39(10): $1278-1284$.

II. Murphy WJ, Eizirik E, O'Brien SJ, Madsen O, Scally M, Douady CJ, Teeling E, Ryder OA, Stanhope MJ, de Jong WW, et al.: Resolution of the early placental mammal radiation using Bayesian phylogenetics. Science 200I, 294(5550):2348-235I.

12. Chen K, Rajewsky N: Natural selection on human microRNA binding sites inferred from SNP data. Nat Genet 2006, 38(I 2): |452-I456.

13. Farh KK, Grimson A, Jan C, Lewis BP, Johnston WK, Lim LP, Burge $\mathrm{CB}$, Bartel DP: The widespread impact of mammalian MicroRNAs on mRNA repression and evolution. Science 2005 , 310(5755): $1817-1821$.

14. Wheeler DL, Barrett T, Benson DA, Bryant SH, Canese $\mathrm{K}$ Chetvernin V, Church DM, DiCuccio M, Edgar R, Federhen S, et al: Database resources of the National Center for Biotechnology Information. Nucleic Acids Res 2006:DI73-180.

15. John B, Enright AJ, Aravin A, Tuschl T, Sander C, Marks DS: Human MicroRNA targets. PLoS Biol 2004, 2 (I I ):e363.

16. Enright AJ, John B, Gaul U, Tuschl T, Sander C, Marks DS: MicroRNA targets in Drosophila. Genome Biol 2003, 5(I):RI.

17. Friedman RC, Farh KK, Burge CB, Bartel DP: Most mammalian mRNAs are conserved targets of microRNAs. Genome Res 2009, I (1):92-105.

18. Baek D, Villen J, Shin C, Camargo FD, Gygi SP, Bartel DP: The impact of microRNAs on protein output. Nature 2008, 455(7209):64-7I.

19. Selbach M, Schwanhausser B, Thierfelder N, Fang Z, Khanin R, Rajewsky $N$ : Widespread changes in protein synthesis induced by microRNAs. Nature 2008, 455(7209):58-63.

20. Subramanian S, Kumar S: Gene expression intensity shapes evolutionary rates of the proteins encoded by the vertebrate genome. Genetics 2004, 168(I):373-38I.

21. Su Al, Wiltshire T, Batalov S, Lapp H, Ching KA, Block D, Zhang J, Soden R, Hayakawa M, Kreiman G, et al.: A gene atlas of the mouse and human protein-encoding transcriptomes. Proc Natl Acad Sci USA 2004, I 0 I (16):6062-6067.

22. Kim SH, Yi SV: Correlated asymmetry of sequence and functional divergence between duplicate proteins of Saccharomyces cerevisiae. Mol Biol Evol 2006, 23(5): 1068-1075.

23. Eisenberg $E$, Levanon $E Y$ : Human housekeeping genes are compact. Trends Genet 2003, I 9(7):362-365.

24. Stark A, Brennecke J, Bushati N, Russell RB, Cohen SM: Animal MicroRNAs confer robustness to gene expression and have a significant impact on 3'UTR evolution. Cell 2005, | 23(6): I | 33-| | 46.

25. Sandberg R, Neilson IR, Sarma A, Sharp PA, Burge CB: Proliferating cells express $m R N A s$ with shortened 3 ' untranslated regions and fewer microRNA target sites. Science 2008, 320(5883): 1643-1647.

26. Gaidatzis D, van Nimwegen E, Hausser J, Zavolan M: Inference of miRNA targets using evolutionary conservation and pathway analysis. BMC Bioinformatics 2007, 8:69.

27. Grimson A, Farh KK, Johnston WK, Garrett-Engele P, Lim LP, Bartel DP: MicroRNA targeting specificity in mammals: determinants beyond seed pairing. Mol Cell 2007, 27(I):9I-I 05.

28. Majoros $\mathrm{WH}$, Ohler U: Spatial preferences of microRNA targets in 3' untranslated regions. BMC Genomics 2007, 8: 152.

29. Bartel DP: MicroRNAs: genomics, biogenesis, mechanism, and function. Cell 2004, I I 6(2):28I-297.

30. Okamura K, Phillips MD, Tyler DM, Duan H, Chou YT, Lai EC: The regulatory activity of microRNA* species has substantial influence on microRNA and 3' UTR evolution. Nat Struct Mol Biol 2008, I 5(4):354-363.

3I. Brockman JM, Singh P, Liu D, Quinlan S, Salisbury J, Graber JH: PACdb: PolyA Cleavage Site and 3'-UTR Database. Bioinformatics 2005, 21(18):369|-3693. 